detected on random biopsy, none were detected with dyespray. Adherence to the 2010 guidelines in these patients was $85 \%$.

1289 individuals with 2256 colonoscopies were identified. Low/moderate/high risk groups were 78 (60\%)/382 (29\%)/125 (9.7\%) respectively. Interval adherence in this cohort was $82 \%$. 20 patients were selected for case notes review. The mean age was 52 (range 31-72). Three patients had colectomy (2 refractory colitis, one DALM) and one patient moved out of area during the studied period. 72 colonoscopies were performed with 52 intervals recorded. Seven (13\%) intervals were scheduled over the recommended time (mean 333 days). These were all at the patient's request. 30 intervals $(58 \%)$ were performed before there intended date (mean -347 days). The most common reason for this was inappropriate yearly surveillance.

Conclusions DALMs were detected in $0.5 \%$ of IBD patients undergoing colonoscopy. Almost half of these were not detected during scheduled surveillance, which may support the shorter intervals used by some clinicians.

\section{PWE-059 TRAVELLING WITH INFLAMMATORY BOWEL DISEASE (IBD): BARRIERS, FEARS, CONCERNS AND SUGGESTIONS FOR SUPPORT}

Anet Soubieres*, Varun Philip, Andrew Poullis. St Georges Hospital NHS Trust, London, UK

\subsection{6/gutjnl-2018-BSGAbstracts. 191}

Introduction IBD can act as a barrier to overseas travel due to concerns about travel related morbidity. This study aims to identify barriers, fears and concerns of IBD patients with regards to travel and possible areas of support from the IBD team.

Method 136 IBD patients were selected using convenience sampling. They completed a questionnaire focused on travel with IBD and specific travel issues (decision to travel, insurance, pre-trip advice, vaccination, previous flares while abroad, high altitude travel, assistance while overseas).

Result 136 patients completed the questionnaire, 70 were male (51.4\%), 73 had Crohn's Disease, 53 had Ulcerative Colitis (UC), 3 had Indeterminate Colitis and 7 were unsure. 56.6\% of patients were taking 5-ASAs, $52.9 \%$ were on immunosuppressant therapy and $20.6 \%$ receiving biologics. $89 \%$ had travelled abroad since their IBD was diagnosed, 30\% reported IBD limited their travel and 40\% said it affected their choice of destination. $61 \%$ worried about healthcare problems abroad. $7 \%$ were refused health insurance and $47 \%$ had travelled abroad uninsured. 9\% travelled uninsured due to their current diagnosis of IBD. Only 64\% felt that they had received adequate pre-travel medical advice. $78 \%$ wanted advice from their doctor for future travel. 60\% were unaware that taking immunosuppressant medication could affect their vaccinations and $63 \%$ on immunosuppressant therapy of biologics claimed they were uninformed of the need to avoid live vaccines. $12 \%$ received live vaccines prior to travel. Suggestions for travel help: $91 \%$ requested a written/electronic prescription for their journey, $75 \%$ wanted specific management advice, $68 \%$ a written management plan. $68 \%$ felt a doctor's note could help them secure health insurance. $38 \%$ of patients who travelled abroad were suffering/recovering from a flare and $18 \%$ travelled despite being within a week of a flare. $92 \%$ were unaware that high altitudes could precipitate a flare. $27 \%$ travelled to high altitude destinations and of these
$46 \%$ experienced a flare whilst travelling or within 4 weeks of travel. $72 \%$ were unaware of the 'Can't Wait Card' and 96\% never heard about the IBD passport website.

Conclusion IBD has a big impact on travel for a broad range of reasons. Refusal of health insurance, coupled with higher premiums could explain why many IBD patients travel abroad uninsured. Providing a doctor's note confirming fitness to travel was a popular solution chosen by patients. A majority were interested in doctors providing written management plans and prescriptions prior to their travel. Despite over half of the study being on immunosuppressant therapy, most were unaware of avoiding live vaccines whilst taking them. Better advice from health care professionals makes this entirely avoidable. Most IBD patients were unaware that high altitudes may precipitate flares. A large majority were unaware of the 'Can't Wait Card' and IBD passport services. Pre-emptive discussions around travel plans should be part of the IBD clinic review.

\section{PWE-060 BASELINE CALPROTECTIN PREDICTS STEROID FREE REMISSION WITH BIOLOGICAL THERAPY IN ULCERATIVE COLITIS AT 1 YEAR}

${ }^{1}$ Sreedhar Subramanian*, ${ }^{2}$ Rebecca Davies, ${ }^{2}$ Susie Dodd, ${ }^{1}$ Daniel Storey, ${ }^{1}$ Alan Steel, ${ }^{1}$ Martyn Dibb, ${ }^{1}$ Paul Collins, ${ }^{1}$ Ed Derbyshire, ${ }^{2}$ Chris Probert, ${ }^{2}$ Paula Mcparland. ${ }^{1}$ Royal Liverpool University Hospital, Liverpool, UK; ${ }^{2}$ University of Liverpool, Liverpool, UK

\subsection{6/gutjnl-2018-BSGAbstracts. 192}

Background Response to the anti-tumour necrosis factor (TNF) agents and the anti-integrin- $\alpha_{4} \beta_{7}$ antibody, vedolizumab, in ulcerative colitis (UC) is variable. There are few clinical predictors of long term efficacy to biological therapy apart from prior exposure to anti-TNF therapy. Identification of readily available clinical and biochemical predictors will enable better utilization of these expensive drugs. We sought to evaluate the role of clinical factors and faecal calprotectin prior to initiation of therapy in predicting steroid free remission at 1 year.

Methods The case records of all patients who commenced biological therapy for UC were examined. Baseline clinical factors including disease extent, duration, smoking status, body mass index, concurrent immunomodulatory or steroid therapy and biochemical factors including C-reactive protein and faecal calprotectin were recorded. Patients with acute severe colitis were excluded. Remission was defined as simple clinical colitis activity index of $<3$. A multi-variate logistic regression was performed to assess the role of baseline variables in predicting steroid free remission at 1 year.

Results A total of 150 patients commenced biological therapy during the study period (2014-2017). Eighteen patients (12\%) were excluded from the final analysis (Adverse reactions, $\mathrm{N}=5,3.3 \%$, Surgery, $\mathrm{N}=6,8 \%$, and data unavailable, $\mathrm{N}=7$, 4.6\%). After exclusion, a total of 98 patients commenced antiTNF therapy (37 infliximab, 14 golimumab and 12 adalimu$\mathrm{mab})$ and 34 commenced vedolizumab. Twenty-three (23.5\%) of the anti-TNF treated and $16(47 \%)$ of vedolizumab treated patients were in steroid free remission at 12 months. Fortyseven patients had extensive colitis and 4 patients were active smokers. A baseline calprotectin of $>500 \mu \mathrm{g} / \mathrm{g}$ was associated with a lower probability of remission to vedolizumab (OR $0.23,95 \%$ CI 0.05 to $0.98, \mathrm{P}<0.047)$ and anti-TNF therapy (OR 0.39 , 95\% CI 0.14 to $1.07, \mathrm{P}=0.067$ ) at 12 months. None of the other clinical variables examined (BMI, disease 